Do We Need a "Coordinating-Emergency Physician" in Mass Casualty Incidents: Four Years Experience (1996-1999) in Bavaria

A. Beck, MD;BM Schneider, MD; H. Gerstacker, MD; L. Kinzl, Prof., $M D$

Department of Trauma Surgery, Hand and Reconstructive Surgery, University Hospital, Ulm, GERMANY

The rescue region of Augsburg, Bavaria, Germany covers a region of $4,100 \mathrm{sq} \mathrm{km}$ with a population of about 823,000 people (city: 265,000; surroundings: 558,000 inhabitants). All of the mass-casualty incidents (MCIs) during which an additional coordinating-emergency physician (CEP) was called have been registered separately since 1996. All 34 events were reviewed (since 1999 and furthermore these events are prospectively registered). There were 19 fire alarms, 6 traffic accidents, 4 poisonings, 3 explosions, 1 mass gathering, and 1 natural disaster.

To gain a good, reliable documentation of quality control efforts, we used some elements of Villareal's quality control modules. In all events, individual emergency medicine could be offered to all patients; none of these patients died due to triage reasons. From these results, we developed a new documentation sheet that facilitates immediate, full, on-scene standardized documentation.

The increasing number of MCls requires specially trained physicians and coordinating rescue to manage these events and provide sufficient prehospital care to all victims. The establishment of official CEP-groups with clearly defined tasks, rules for liability, and fields of competence will help to guarantee the provision of individual emergency medicine to all victims. Excellent documentation is needed not only for quality reasons, but it also is required for future analysis of each event.

Keywords: complex emergencies; coordinating emergency physician; disaster medicine; mass casualties

E-mail: alexander.beck@medizin.uni-ulmde

\section{Work of the Hospital of ARCDM "Zaschita" in Earthquakes}

I.A. Nazarova, A.V. Garkavi

All-Russian Centre for Disaster Medicine "Zaschita", Moscow Medical Academy, Moscow, RUSSIA

The experience with relief following great earthquakes in different places (Sakhalin Island, 1995; Columbia, 1999; Turkey, 1999) indicates that during rescue efforts, the number of the injured extricated alive from the ruins increases considerably by the 3 th-4th days, but, by the 5 th-6th days, practically no injured alive victims can be delivered for medical care.

Thus, when deploying a hospital at the disaster site during the first 3-4 days, those extricated from the ruins prevail among the injured, and in the structure of injuries, crush syndrome predominates (47.4\%). In this situation, the priority task is quick evacuation of the injured to a specialized, in-patient hospital for carrying out full detoxification therapy. To define the kind of medical assistance required, it is necessary to minimize the medical-diagnostic manipulations as priority is directed at anti-shock measures.

By the 4th-5th days, isolated injuries of locomotor system (non-penetrating wounds, isolated fractures, closed injuries of soft tissues) prevail in the injuries structure (up to $70 \%$ ). The majority of these injured do not need inpatient treatment. When the work of local public health function is broken, the tasks of the hospital are not to decrease, but to enlarge medical assistance - to the delivery of specialized care (updated radio-diagnosis of injuries, surgical treatment of infected wounds, repositioning of fractures with plaster casts, percutaneous and out-locus fixation).

Thus, in rendering medical care following earthquakes, we may mark out two temporal periods that differ by the cohorts of the injured and structure of injuries. Timely maneuvers with the type of medical care promotes increasing efficiency of the work.

Keywords: characteristics; crush syndrome; detoxification; earthquake; hospitals; periods; responses

Email: rcdm.org@g23.relcom.ru 